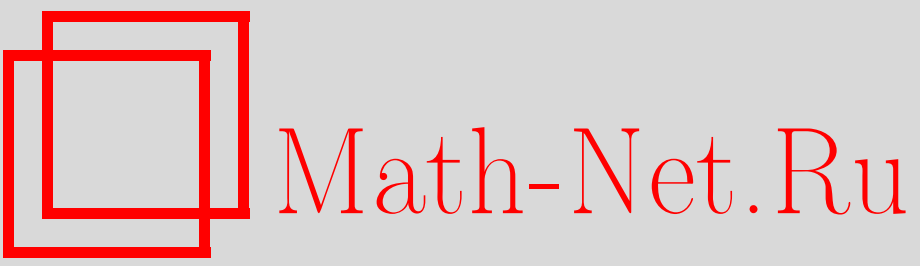

Ю. Кодама, Топология вещественной части гиперэллиптического якобиана, связанного с периодической решеткой Тоды, ТМФ, 2002, том 133, номер 3, 439-462

DOI: https://doi.org/10.4213/tmf410

Использование Общероссийского математического портала Math-Net.Ru подразумевает, что вы прочитали и согласны с пользовательским соглашением

http://www.mathnet.ru/rus/agreement

Параметры загрузки:

IP: 35.173 .219 .12

26 апреля 2023 г., 06:43:03 
ТЕОРЕТИЧЕСКАЯ

И МАТЕМАТИЧЕСКАЯ

ФИЗИКА

Том 133, № 3

декабрь, 2002

(C) 2002 r.

Ю. Кодама*

\section{ТОПОЛОГИЯ ВЕЩЕСТВЕННОЙ ЧАСТИ ГИПЕРЭЛЛИПТИЧЕСКОГО ЯКОБИАНА, СВЯЗАННОГО С ПЕРИОДИЧЕСКОЙ РЕШЕТКОЙ ТОДЫ}

Исследуется топология изоспектрального вещественного многообразия $\mathfrak{s l}(N)$-периодической решетки Тоды, состоящей из $2^{N-1}$ различных систем. Решения этих систем имеют сингулярные точки, множество которых определяет дивизор на многообразии. При добавлении дивизора многообразие компактифицируется как вещественная часть $(N-1)$-мерного многообразия Якоби, связанного с гиперэллиптической римановой поверхностью рода $g=N-1$. Изучена вещественная структура дивизора и высказаны гипотезы о топологии аффинной части вещественного якобиана и о правиле склейки по дивизору для компактификации многообразия на основе знакового представления группы Вейля $\mathfrak{s l}(N)$.

Ключевые слова: периодическая решетка Тоды, многообразие Якоби, тета-дивизор, тетафункция Римана.

\section{1. ВВЕДЕНИЕ}

Бесконечная решетка Тоды определяется как гамильтонова система с гамильтониаHOM

$$
H=\frac{1}{2} \sum_{k \in \mathbb{Z}} P_{k}^{2}+\sum_{k \in \mathbb{Z}} \epsilon_{k} e^{Q_{k}-Q_{k+1}},
$$

где $\epsilon_{k} \in\{ \pm 1\}$. Решение этой системы сингулярно, если по крайней мере один из знаков $\epsilon_{k}$ в потенциальном члене является отрицательным [1]. При использовании координат $\Phi$ лашки $a_{k}:=\epsilon_{k} e^{Q_{k}-Q_{k+1}}$ и $b_{k}:=-P_{k}$ уравнения Гамильтона с гамильтонианом $H$ имеют вид

$$
\begin{aligned}
\frac{d a_{k}}{d t} & =a_{k}\left(b_{k+1}-b_{k}\right), \\
\frac{d b_{k}}{d t} & =a_{k}-a_{k-1} .
\end{aligned}
$$

* Department of Mathematics, Ohio State University, Columbus, USA.

E-mail: kodama@math.ohio-state.edu 
ЗАмечАниЕ 1.1. Знак $\epsilon_{k}$ инвариантен относительно потока Тоды, и аффинная часть изоспектрального многообразия потока Тоды состоит из $2^{N-1}$ несвязных кусков, занумерованных знаками $a_{k}$ (заметим, что величина $\prod_{k=1}^{N} a_{k}$ является инвариантом).

Бесконечная решетка Тоды для функций $\left\{\left(a_{k}, b_{k}\right): k \in \mathbb{Z}\right\}$ может быть приведена к матричному уравнению - формулировке Лакса

$$
\frac{d L}{d t}=[L, A]
$$

где $L-(\infty \times \infty)$-матрица вида

$$
L=\left(\begin{array}{cccccccccc}
\ddots & \ddots & \ddots & \ddots & & & & & & \\
\ddots & a_{-1} & b_{0} & 1 & 0 & & & & & \\
& 0 & a_{0} & b_{1} & 1 & 0 & & & & \\
& & \ddots & \ddots & \ddots & \ddots & \ddots & & & \\
& & & & 0 & a_{k-2} & b_{k-1} & 1 & 0 & \\
& & & & & 0 & a_{k-1} & b_{k} & 1 & \ddots \\
& & & & & & \ddots & \ddots & \ddots & \ddots
\end{array}\right)
$$

а $A$ - строго нижнетреугольная часть $L$, т.е. $A=(L)$ _. Уравнение Лакса есть в точности условие совместности следуюших линейных уравнений при условии изоспектральности:

$$
\begin{aligned}
L \phi & =\lambda \phi, \\
\frac{d \phi}{d t} & =A \phi .
\end{aligned}
$$

$\mathfrak{s l}(N)$-периодическая решетка Тоды задается условиями $Q_{k+N}=Q_{k}$ и $P_{k+N}=P_{k}$, из которых следует, что

$$
a_{k+N}=a_{k}, \quad b_{k+N}=b_{k} .
$$

Тогда собственный вектор $\phi=\left(\ldots, \phi_{0}, \phi_{1}, \phi_{2}, \ldots\right)^{\mathrm{T}}$ удовлетворяет уравнению

$$
\phi_{k+N}=z \phi_{k},
$$

где $z$-спектральный параметр, являющийся собственным значением монодромии этой периодической задачи. Матрица Лакса для $\mathfrak{s l}(N)$-периодической решетки Тоды имеет вид

$$
L_{P}=\left(\begin{array}{ccccccc}
b_{1} & 1 & 0 & \cdots & \cdots & \cdots & a_{N} z^{-1} \\
a_{1} & b_{2} & 1 & \cdots & \cdots & \cdots & 0 \\
\vdots & \ddots & \ddots & \ddots & \ddots & \ddots & \vdots \\
0 & \cdots & \cdots & \cdots & a_{N-2} & b_{N-1} & 1 \\
z & \cdots & \cdots & \cdots & \cdots & a_{N-1} & b_{N}
\end{array}\right) .
$$


Характеристическое уравнение для $L_{P}$ определяет алгебраическую кривую

$$
\operatorname{det}\left(L_{P}-\lambda I\right)=-\left(z+\frac{\prod_{i=1}^{N} a_{i}}{z}-P(\lambda)\right)=0
$$

которая представляет собой одномерное аффинное многообразие на $(\lambda, z) \in \mathbb{C}^{2}$. Здесь $P(\lambda)-N$-й многочлен от $\lambda$ вида

$$
P(\lambda):=\Delta_{1, N}(\lambda)-a_{N} \Delta_{2, N-1}(\lambda)
$$

где $\Delta_{m, n}(\lambda)$ при $n \geqslant m$ определяется как

$$
\Delta_{m, n}(\lambda):=\left|\begin{array}{ccccccc}
b_{m}-\lambda & 1 & 0 & \cdots & \cdots & \cdots & 0 \\
a_{m} & b_{m+1}-\lambda & 1 & \cdots & \cdots & \cdots & 0 \\
\vdots & \ddots & \ddots & \ddots & \ddots & \ddots & \vdots \\
0 & \ldots & \ldots & \cdots & a_{n-2} & b_{n-1}-\lambda & 1 \\
0 & \ldots & \cdots & \cdots & 0 & a_{n-1} & b_{n}-\lambda
\end{array}\right|
$$

Мы полагаем, что все корни дискриминанта уравнения (1.8)

$$
F(\lambda):=P(\lambda)^{2}-4 \prod_{j=1}^{N} a_{j}
$$

различны, т.е.

$$
F(\lambda)=\prod_{k=1}^{2 N_{R}}\left(\lambda-\lambda_{k}\right) \prod_{l=1}^{N_{I}}\left(\left(\lambda-\nu_{l}\right)\left(\lambda-\bar{\nu}_{l}\right)\right),
$$

где все $\lambda_{j} \in \mathbb{R}, \nu_{l} \in \mathbb{C}$ различны и $N_{R}+N_{I}=N$. Отсюда следует, что алгебраическая кривая, для которой выполняется это условие, является гладким аффинным многообразием. Будем говорить, что матрица $L_{P}$ находится в классе $(\mathrm{P})$, если соответствующая матрица периодов $L$ удовлетворяет этому условию. Фазовое пространство периодической решетки имеет вид

$$
Z_{\mathbb{R}}^{P}:=\left\{\left(a_{1}, \ldots, a_{N}, b_{1}, \ldots, b_{N}\right) \in \mathbb{R}^{2 N}: L_{P} \quad \text { в классе }(\mathrm{P})\right\}
$$

Поскольку $\mathfrak{s l}(N)$-решетка Тоды является гамильтоновой системой степени $N$, ее интегрируемость можно доказать, найдя $N$ независимых инволютивных интегралов. Эти интегралы $I_{k}$ даются коэффициентами характеристического уравнения $(1.8)$, где $P(\lambda)$ определяется как

$$
P(\lambda)=(-1)^{N}\left(\lambda^{N}+\sum_{k=1}^{N}(-1)^{k} I_{k}\left(L_{P}\right) \lambda^{N-k}\right)
$$


и имеется еше дополнительный интеграл $\prod_{i=1}^{N} a_{i}$, получаемый из вычета по спектральному параметру $z$ :

$$
\begin{aligned}
I_{1}\left(L_{P}\right) & =\sum_{i=1}^{N} b_{i}, \\
I_{2}\left(L_{P}\right) & =\sum_{i>j} b_{i} b_{j}-\sum_{i=1}^{N} a_{i}, \\
\ldots \ldots \ldots \ldots \ldots \ldots \ldots \ldots \ldots \ldots \ldots \ldots \ldots \ldots \ldots \ldots \ldots \ldots & \ldots \ldots \ldots \\
I_{N}\left(L_{P}\right) & =\Delta_{1, N}(0)-a_{N} \Delta_{2, N-1}(0)=\prod_{i=1}^{N} b_{i}+\cdots, \\
I_{N+1}\left(L_{P}\right) & =\prod_{i=1}^{N} a_{i} .
\end{aligned}
$$

Тогда изоспектральное множество, которое определяется соотношением

$$
Z_{\mathbb{R}}^{P}(\gamma):=\left\{\left(a_{1}, \ldots, a_{N}, b_{1}, \ldots, b_{N}\right) \in Z_{\mathbb{R}}^{P}: I_{k}\left(L_{P}\right)=\gamma_{k} \in \mathbb{R}, k=1, \ldots, N+1\right\},
$$

представляет собой аффинную часть компактифицированного многообразия $\widehat{Z}_{\mathbb{R}}^{P}(\gamma)$ размерности $\operatorname{dim} Z_{\mathbb{R}}^{P}(\gamma)=N-1$, а с учетом дивизора $\Theta$, связанного с сингулярностями $a_{k}$, $k=1, \ldots, N$, мы имеем $[2]$

$$
Z_{\mathbb{R}}^{P}(\gamma)=\widehat{Z}_{\mathbb{R}}^{P}(\gamma) \backslash \Theta, \quad \Theta=\bigcup_{k=1}^{N}\left\{a_{k}^{-1}=0\right\}
$$

Оказывается, что компактное многообразие $\widehat{Z}_{\mathbb{R}}^{P}(\gamma)$ можно отождествить с вешественной частью якобиана $\mathbb{C}^{g} / \Gamma$, где решетка $\Gamma$ определена с помошью матрицы периодов $\Omega$, связанной с римановой поверхностью рода $g$ (подробнее см. следующий раздел). Риманова поверхность определяется спектральной кривой, и ее род $g=N-1$. Мы обсудим некоторые детали этого утверждения в следующих разделах. Главная цель настоящей работы состоит в нахождении топологической структуры дивизора $\Theta$ в вещественной части якобиана и в определении правила склейки на дивизоре для компактифицирования афффинного многообразия $Z_{\mathbb{R}}^{P}(\gamma)$. Предварительно в разделе 2 кратко опишем некоторые свойства алгебраических кривых и решения периодической решетки Тоды.

\section{2. АЛГЕБРАИЧЕСКИЕ КРИВЫЕ И ПЕРИОДИЧЕСКИЕ РЕШЕТКИ ТОДЫ}

2.1. Риманова поверхность и якобиан. Характеристическое уравнение $\operatorname{det}\left(L_{P}-\lambda I\right)=0$ определяет вешественную плоскую кривую $\mathcal{C}$ (одномерную аффииную кривую на $\mathbb{C}^{2}$ ) как

$$
y^{2}=P(\lambda)^{2}-4=\prod_{k=1}^{2 N}\left(\lambda-\lambda_{k}\right)
$$


где сделана замена координат $(\lambda, z)$ на $(\lambda, y)$ при $y=2 z+P(\lambda)$. Обозначим комплексно-сопряженные пары корней как $\left(\lambda_{N_{R}+2 k-1}=\nu_{k}, \lambda_{N_{R}+2 k}=\bar{\nu}_{k}\right)$ при $k=1, \ldots, N_{I}$, как в формуле (1.10). Тогда компактная риманова поверхность, связанная с плоской кривой $\mathcal{C}$, получается добавлением точек на бесконечности путем введения второй карты $\mathcal{C}^{\prime}$,

$$
\left(y^{\prime}\right)^{2}=\prod_{k=1}^{2 N}\left(1-\lambda_{k} \lambda^{\prime}\right),
$$

которая склеивается с картой $\mathcal{C}$ с помошью изоморфизма $\lambda^{\prime}=1 / \lambda, y^{\prime}=y / \lambda^{N}$. Эта риманова поверхность $\mathcal{R}=\mathcal{C} \cup \mathcal{C}^{\prime}$ называется гиперэллиптической римановой поверхностью рода $g=N-1$ и топологически является компактной поверхностью с $g$ ручками. На $\mathcal{R}$ можно определить морфизм $\pi: \mathcal{R} \rightarrow \mathbb{C} P^{1}$, полагая $(\lambda, y) \mapsto \lambda$ на карте $\mathcal{C}$ и аналогично на карте $\mathcal{C}^{\prime}$. Отметим, что морфизм $\pi$ является двукратным накрытием $\mathbb{C}^{1}$, за исключением точек ветвления $\lambda_{k}$. Точки на бесконечной части поверхности $\mathcal{R}$, обозначаемые как $p_{\infty}^{ \pm}$, суть $\left(\lambda^{\prime}=0, y^{\prime}= \pm 1\right)$, т.е.

$$
\mathcal{R}=\mathcal{R}_{0} \cup\left\{p_{\infty}^{+}, p_{\infty}^{-}\right\}
$$

где $\mathcal{R}_{0}$ - аффинная часть поверхности $\mathcal{R}$. Можно также определить симплектический базис в группе $H_{1}(\mathcal{R}, \mathbb{Z})$, обозначаемый как $\left\{\left(\alpha_{j}, \beta_{j}\right): j=1, \ldots, g\right\}$, где пути $\alpha_{i}$ и $\alpha_{j}$, так же как и пути $\beta_{i}$ и $\beta_{j}$, не пересекаются друг с другом, тогда как пути $\alpha_{i}$ и $\beta_{j}$ пересекаются, причем только в одной точке, где $i=j$.

Определим теперь вещественную часть римановой поверхности, обозначаемую $\mathcal{R}_{\mathbb{R}}$, как множество фиксированных точек антиголоморфной инволюции, получаемой путем сопряжения $(\lambda, y) \mapsto(\bar{\lambda}, \bar{y})$ :

$$
\mathcal{R}_{\mathbb{R}}:=\{(\lambda, y) \in \mathcal{R}: \lambda=\bar{\lambda}, y=\bar{y}\} .
$$

Тогда легко видеть, что имеет место

ПРЕДЛОЖЕНИЕ 2.1. Пусть $\mathcal{R}$ - компактная (гладкая) риманова поверхность рода $g$. Тогда ее вещественная часть $\mathcal{R}_{\mathbb{R}}$ изоморфна несвязному обвединению $N_{R}$ окружностей, $\mathbb{R} P^{1} \cong S^{1}$ :

$$
\mathcal{R}_{\mathbb{R}} \cong \overbrace{S^{1} \sqcup \cdots \sqcup S^{1}}^{N_{R}},
$$

əде $2 N_{R}$ - число вещественных нулей в уравнении (2.1), т.е. вещественных точек ветвления поверхности $\mathcal{R}$.

Согласно теореме Римана-Роха на римановой поверхности $\mathcal{R}$ рода $g$ имеются $g$ голоморфных дифференциалов, которые мы обозначим как $\omega_{1}, \ldots, \omega_{g}$. Нормируем эти дифференциалы условиями

$$
\oint_{\alpha_{i}} \omega_{j}=\delta_{i, j}, \quad i, j=1, \ldots, g
$$


Тогда связанный с $\mathcal{R}$ якобиан $\operatorname{Jac}(\mathcal{R})$ определяется как комплексный тор с решеткой $\Gamma$ ранга $2 g$ :

$$
\operatorname{Jac}(\mathcal{R})=\mathbb{C}^{g} / \Gamma, \quad \Gamma:=\left\{\Omega m: m=\left(m_{1}, \ldots, m_{2 g}\right)^{\mathrm{t}} \in \mathbb{Z}^{2 g}\right\},
$$

где $(g \times 2 g)$-матрица $\Omega$ - матрица периодов, определяемая как

$$
\Omega=(I, B), \quad B=\left.\left(\oint_{\beta_{i}} \omega_{j}\right)\right|_{1 \leqslant i, j \leqslant g},
$$

где $I$ - единичная $(g \times g)$-матрица. Оказывается, что наша цель - исследования топологической структуры изоспектрального многообразия периодической решетки Тоды сводится именно к изучению топологической структуры вещественной части якобиана и его дивизоров, соответствующих сингулярностям потока Тоды.

2.2. Периодическая решетка Тоды. Поток Тоды можно описывать как линейньй поток на якобиане $\operatorname{Jac}(\mathcal{R})$ римановой поверхности $\mathcal{R}$. Сначала получим решение в простейшем случае $N=2(g=1)$, а общий случай обсудим в следующем разделе. Матрица Лакса $L_{P}$ в этом случае имеет вид

$$
L_{P}=\left(\begin{array}{cc}
b_{1} & 1+a_{2} z^{-1} \\
z+a_{1} & b_{2}
\end{array}\right)
$$

а ее спектральная кривая есть эллиптическая кривая

$$
\operatorname{det}\left(L_{P}-\lambda I\right)=-\left(z+\frac{a_{1} a_{2}}{z}-P(\lambda)\right)=0
$$

Полином $P(\lambda)$ имеет вид

$$
P(\lambda)=\lambda^{2}+I_{2}, \quad I_{2}=-b_{1}^{2}-a_{1}-\frac{1}{a_{1}}
$$

и мы полагаем, что $I_{1}=b_{1}+b_{2}=0$ и $I_{3}=a_{1} a_{2}=1$. Тогда аффинная часть изоспектрального многообразия имеет вид

$$
Z_{\mathbb{R}}^{P}(\gamma)=\left\{\left(a_{1}, b_{1}\right) \in \mathbb{R}^{2}: b_{1}^{2}+a_{1}+\frac{1}{a_{1}}=\gamma_{2} \in \mathbb{R}\right\} \cong \begin{cases}S^{1} \sqcup \mathbb{R} \sqcup \mathbb{R}, & \gamma_{2}>2 \\ \mathbb{R} \sqcup \mathbb{R}, & \gamma_{2}<2\end{cases}
$$

Таким образом, имеем

$$
Z_{\mathbb{R}}^{P}(\gamma) \cong \mathcal{R}_{\mathbb{R}} \backslash\left\{p_{\infty}^{+}, p_{\infty}^{-}\right\}=\mathcal{R}_{\mathbb{R}} \cap \mathcal{R}_{0}
$$

Два несвязных куска $\mathbb{R}$ компактифицированы так, что образуют окружность $S^{1}$, т.е. соответствуюшее решение становится сингулярным один раз в каждой точке $p_{\infty}^{+}$или $p_{\infty}^{-}$ 
и в целом дважды за один цикл. Таким образом, компактифицированное многообразие $\widehat{Z}_{\mathbb{R}}^{P}(\gamma)$ есть в точности $\mathcal{R}_{\mathbb{R}}$ (см. более точное утверждение ниже).

Поток Тоды определяется уравнениями

$$
\frac{d a_{1}}{d t}=-2 a_{1} b_{1}, \quad \frac{d b_{1}}{d t}=a_{1}-\frac{1}{a_{1}}
$$

решение которых можно получить, исключая $a_{1}$ из (2.5):

$$
\frac{d b_{1}}{d t}= \pm \sqrt{\left(b_{1}^{2}+I_{2}\right)^{2}-4}
$$

что можно также записать в виде

$$
\left.\frac{d \lambda}{y(\lambda)}\right|_{\lambda=b_{1}}= \pm d t
$$

Здесь $y(\lambda)=\sqrt{\left(\lambda^{2}+I_{2}\right)^{2}-4}$ и дифференциал $\omega_{1}=d \lambda / y$ является голоморфным дифференциалом на $\mathcal{R}$. Тогда решение можно записать с помощью әллиптического интеграла

$$
\int_{b_{0}}^{b_{1}} \omega_{1}= \pm t+\delta_{1},
$$

где $b_{0}$ - фиксированная точка, а $\delta_{1}$ - произвольная константа. Поскольку интеграл (2.7) зависит от пути из $b_{0}$ в $b_{1}$, его следует определять на якобиане $\operatorname{Jac}(\mathcal{R})$ - комплексном торе $\mathbb{C} / \Gamma$ с решеткой $\Gamma$, порожденной матрицей периодов $\Omega=\left(1, B_{11}\right)$, где $B_{11}=\oint_{\beta_{1}} \omega_{1}$. Более того, эллиптический интеграл (2.7) должен определяться на вешественной части якобиана. А именно, мы имеем следующие отображения с использованием отображения Абеля-Якоби $v$ для описания потока Тоды:

$$
\begin{aligned}
Z_{\mathbb{R}}^{P}(\gamma) & \longrightarrow \mathcal{R}_{\mathbb{R}} \stackrel{v}{\longrightarrow} \operatorname{Jac}(\mathcal{R})_{\mathbb{R}}, \\
b_{1} & \longmapsto \mu_{1} \longmapsto\left[\int_{\mu_{*}}^{\mu_{1}} \omega_{1}\right],
\end{aligned}
$$

где $\mu_{*}$ - фиксированная точка на вешественной части римановой поверхности $\mathcal{R}_{\mathbb{R}}$. Отсюда следует, что поток Тоды линеен на якобиане, и решение $b_{1}(t)$ дается решением задачи обрашения Якоби (в данном случае в точности эллиптической функцией Якоби), которая определяется как обратное отображение к $v: \psi: \operatorname{Jac}(\mathcal{R}) \longrightarrow \mathcal{R}$. Тогда решение задачи обрашения Якоби определяется нулями тета-функции Римана (явный вид решения для потока Тоды в случае рода $g>1$ приведен в следуюшем разделе). Теперь задача состоит в определении структуры вешественной части $\operatorname{Jac}(\mathcal{R}) \backslash \Theta$, изоморфной $Z_{\mathbb{R}}^{P}(\gamma)$. В этом примере дивизор $\Theta$ имеет вид

$$
\Theta=\left\{v\left(p_{\infty}^{+}\right), v\left(p_{\infty}^{-}\right)\right\}
$$


а компактифицированное многообразие есть $\widehat{Z}_{\mathbb{R}}^{P}(\gamma) \cong \mathcal{R}_{\mathbb{R}} \cong \operatorname{Jac}(\mathcal{R})_{\mathbb{R}}$, т.е.

$$
\operatorname{Jac}(\mathcal{R})_{\mathbb{R}} \cong \begin{cases}S^{1} \times \mathbb{Z} / 2 \mathbb{Z}, & \gamma_{2}>2, \\ S^{1}, & \gamma_{2}<2,\end{cases}
$$

где каждая окружность $S^{1}$ занумерована знаками $\left(\epsilon_{1}, \epsilon_{2}\right)$, т.е. $(++)$ или $(--)$ при $\gamma_{2}>2$ и $(--)$ при $\gamma_{2}<2$. Таким образом, одна из окружностей $S^{1}$ при $\gamma_{2}>2$ получается склейкой двух копий $\mathbb{R}$ на $\Theta$. Нахождение структуры склейки аффинных кусков вещественной части якобиана $\operatorname{Jac}(\mathcal{R})_{\mathbb{R}} \backslash \Theta \cong Z_{\mathbb{R}}^{P}(\gamma)$ является одной из основных целей настоящей работы.

Для случая произвольного рода достаточно давно известно, что число связных компонент в вешественной части якобиана, обозначаемых как $n\left(\mathrm{Jac}_{\mathbb{R}}\right):=$ $\operatorname{rank} H_{0}\left(\operatorname{Jac}(\mathcal{R})_{\mathbb{R}}, \mathbb{Z}\right)$, полностью определяется числом связных компонент в вешественной части римановой поверхности $N_{R}=\operatorname{rank} H_{0}\left(\mathcal{R}_{\mathbb{R}}, \mathbb{Z}\right)$, т.е. справедливо

ПрЕДЛОЖЕНИЕ 2.2 ([3], см. также [4]). Пусть $\mathcal{R}$ - компактная риманова поверхность рода g с непустой вещественной компонентой, $N_{R} \neq 0$. Тогда кажсдая связная компонента $\operatorname{Jac}(\mathcal{R})_{\mathbb{R}}$ изоморфна $g$-мерному вещественному тору $\mathbb{T}^{g}=$ $\mathbb{R}^{g} / \mathbb{Z}^{g} u$

$$
\operatorname{Jac}(\mathcal{R})_{\mathbb{R}} \cong \mathbb{T}^{g} \times(\mathbb{Z} / 2 \mathbb{Z})^{m},
$$

әде $m=N_{R}-1 \leqslant g$, и, таким образом, $n\left(\mathrm{Jac}_{\mathbb{R}}\right)=2^{m}$.

Доказательство этого утверждения, основанное на структуре решения периодической решетки Тоды, дано в следуюшем разделе. Далее мы изучим топологическую структуру аффинных частей $\mathrm{Jac}(\mathcal{R})_{\mathbb{R}}$ и дивизор $\Theta$ и найдем правило склейки аффинных частей, занумерованных знаками $\left(\epsilon_{1}, \ldots, \epsilon_{N}\right)$ для компактификации. В работе [2] было показано, что дивизор $\Theta$ состоит из $g+1$ неприводимых компонент $\Theta_{k}$, где $a_{k}$ имеет сингулярность (см. (1.12)). Тогда можно ожидать, что каждый тор $\mathbb{T}^{g}$ в предложении 2.2 поделен на $g+1$ афффинных многообразий с помошью этих дивизоров $\Theta_{k}$. Для того чтобы изучить случай произвольного рода, в разделе 3 мы дадим краткий обзор теории периодической решетки Тоды и формулу для решения в терминах тета-функции Римана $\vartheta(x \mid B)$. Они используются в разделе 4 при изучении геометрии дивизоров, которые даются нулями $\vartheta(x \mid B)$ с некоторыми трансляциями по $x$.

\section{3. РЕШЕНИЕ ПЕРИОДИЧЕСКОЙ РЕШЕТКИ ТОДЫ}

3.1. Изоспектральное многообразие и симметрические произведения римановых поверхностей. Пусть пара бесконечномерных векторов $\left\{\phi^{(1)}, \phi^{(2)}\right\}$ является фундаментальным набором решений разностного уравнения $L \phi=\lambda \phi$ с условием периодичности (1.5). Предполагается, что векторы $\phi^{(j)}=\left(\ldots, \phi_{-1}^{(j)}, \phi_{0}^{(j)}, \phi_{1}^{(j)}, \ldots\right)$ удовлетворяют соотношениям

$$
\begin{array}{ll}
\phi_{0}^{(1)}=1, & \phi_{1}^{(1)}=0 \\
\phi_{0}^{(2)}=0, & \phi_{1}^{(2)}=1 .
\end{array}
$$


(Отметим, что выбранный индекс "0" можно сдвигать на произвольное число $k$ $(\bmod N+1)$.$) Тогда можно определить матрицу монодромии M$, полагая

$$
\left(\begin{array}{c}
\phi_{k+N}^{(1)} \\
\phi_{k+N}^{(2)}
\end{array}\right)=M\left(\begin{array}{c}
\phi_{k}^{(1)} \\
\phi_{k}^{(2)}
\end{array}\right), \quad M=\left(\begin{array}{cc}
\phi_{N}^{(1)} & \phi_{N+1}^{(1)} \\
\phi_{N}^{(2)} & \phi_{N+1}^{(2)}
\end{array}\right) .
$$

Лемма 3.1. При условии $\prod_{k=1}^{N} a_{k}=1$ имеем $\operatorname{det} M=1$.

ДокАЗАТЕльСТво. Легко показать, что вронскиан $W(n):=\phi_{n}^{(1)} \phi_{n+1}^{(2)}-\phi_{n}^{(2)} \phi_{n+1}^{(1)}$ удовлетворяет соотношению

$$
W(n)=a_{n-1} W(n-1)=\left(\prod_{k=0}^{n-1} a_{k}\right) W(0) .
$$

Положим тогда $n=N$, т.е. $W(N)=\operatorname{det} M$, и используем равенство $W(0)=1$ для завершения доказательства.

Из условия периодичности (1.6) можно заключить, что спектральный параметр $z$ является собственным значением $M$, и мы получим

$$
z+\frac{1}{z}-\operatorname{tr} M=0
$$

Тогда справедливо равенство

$$
\operatorname{tr} M=\phi_{N}^{(1)}+\phi_{N+1}^{(2)}=P(\lambda),
$$

где $N$-й полином $P(\lambda)$ дается соотношением (1.11).

Найдем явный вид фундаментальных решений $\phi^{(j)}$. Путем прямого вычисления легко показать, что справедлива

ЛЕмма 3.2. При $n \geqslant 1$ величинь $\phi_{n+1}^{(j)}$ даются соотношениями

$$
\phi_{n+1}^{(1)}=-a_{N}(-1)^{n-1} \Delta_{2, n}, \quad \phi_{n+1}^{(2)}=(-1)^{n} \Delta_{1, n},
$$

где $\Delta_{m, n}$ определяются формулой (1.9), а $\Delta_{2,1}:=1$.

Рассмотрим теперь так называемый вспомогательный спектр $\mu_{n}$, определяемый корнями уравнения $\phi_{N+1}^{(1)}(\lambda)=0$. Обозначим

$$
u_{g}(\lambda):=(-1)^{N-1} \Delta_{2, N}(\lambda)=\prod_{k=1}^{N-1}\left(\lambda-\mu_{k}\right)=0,
$$

где $g=N-1$. Отметим, что некоторые из корней являются комплексными и образуют пару комплексно-сопряженных корней $\left(\mu_{k}, \bar{\mu}_{k}\right)$. С учетом (3.5) решения решетки Тоды $a_{k}$ и $b_{k}$ выражаются через симметрические функции корней $\mu_{j}$ при условиях изоспектральности $I_{k}=\gamma_{k}$ при $k=1, \ldots, N+1$. Например, из определения (3.5) и с учетом равенства $I_{1}\left(L_{P}\right)=0$ имеем

$$
b_{1}=-\sum_{j=2}^{N} b_{j}=-\sum_{n=1}^{N-1} \mu_{n} .
$$

Отсюда следует 
ПРЕДЛОЖЕНИЕ 3.1. Отображсние $\varphi$ из $Z_{\mathbb{R}}^{P}(\gamma)$ в вещественную часть $g$-кратного симметрического произведения аффинной части $\mathcal{R}_{0}$, обозначаемую как $\mathcal{R}_{0}^{(g)}:=$ $\mathcal{R}_{0}^{g} / S_{g}$,

$$
\varphi: Z_{\mathbb{R}}^{P}(\gamma) \longrightarrow\left(\mathcal{R}_{0}^{(g)}\right)_{\mathbb{R}}
$$

является сюрвективным. Здесь $S_{g}$ - симметрическая группа порядка $g=N-1$.

Формулировка решетки Тоды с переменными $\mu_{k}$ называется системой Мамфорда [5]; она параметризует пространство модулей гиперэллиптических римановых поверхностей через тройки полиномов $\left(u_{g}(\lambda), v_{g}(\lambda), w_{g}(\lambda)\right)$, определяемых факторизацией кривой $(2.1)$ :

$$
y^{2}=u_{g}(\lambda) w_{g}(\lambda)+v_{g}^{2}(\lambda)
$$

Здесь $u_{g}(\lambda)$ дается формулой $(3.5), v_{g}(\lambda)$ - полином степени $g-1=N-2$, а $w_{g}(\lambda)-$ приведенньй (т.е. с единичным коэффициентом при старшем члене) полином степени $g+2$. Отметим, что если все корни $\mu_{k}$ различны, то $v_{g}(\lambda)$ можно выразить в терминах $\mu_{k}$ при условии $v_{g}\left(\mu_{k}\right)=y\left(\mu_{k}\right)$, т.е.

$$
v_{g}(\lambda)=\sum_{k=1}^{g} y\left(\mu_{k}\right) \prod_{j \neq k} \frac{\lambda-\mu_{j}}{\mu_{k}-\mu_{j}}
$$

Тройки системы Мамффорда $\left(u_{d}(\lambda), v_{d}(\lambda), w_{d}(\lambda)\right)$ при $0<d<g$ можно также использовать для параметризации дивизоров (см. следующий раздел).

Обсудим теперь топологию множества корней $\mu_{k}$. Прежде всего отметим, что $\pi\left(\mathcal{R}_{\mathbb{R}}\right)$ (где $P=(\lambda, y) \mapsto \pi(P)=\lambda$ ) состоит из нескольких интервалов

$$
\pi\left(\mathcal{R}_{\mathbb{R}}\right)=\bigcup_{m=0}^{N_{R}} \Lambda_{m}, \quad 1 \leqslant N_{R} \leqslant N
$$

где $\Lambda_{m}=\left[\lambda_{2 m}, \lambda_{2 m+1}\right]$ при $\lambda_{0}=\lambda_{2 N_{R}+1}=\infty$ (т.е. $\left[\lambda_{0}, \lambda_{1}\right]$ и $\left[\lambda_{2 N_{R}}, \lambda_{2 N_{R}+1}\right]$ связаны через $\left.\pi\left(p_{\infty}^{ \pm}\right)\right)$.

ЛЕмМа 3.3. Если корень $\mu_{k}$ является вещественным, то он принадлежсит одному из интервалов $\Lambda_{m}$

ДокАЗАТЕльство. Поскольку $\phi_{N+1}^{(1)}\left(\mu_{k}\right)=0$, имеем

$$
\operatorname{tr} M=\phi_{N}^{(1)}+\frac{1}{\phi_{N}^{(1)}}
$$

Отсюда следует, что $|\operatorname{tr} M| \geqslant 2$, а потому $\mu_{k} \in \pi\left(\mathcal{R}_{\mathbb{R}}\right)$.

Поскольку множество точек $P_{k} \in \mathcal{R}$ состоит из вешественных и/или пары комплексно-сопряженных точек, имеем следующую лемму о топологии этих множеств. 
Лемма 3.4 [5]. Множсество точек $P_{k} \in \mathcal{R}$ таких, что $\pi\left(P_{k}\right)=\mu_{k}, k=1, \ldots, g$, можно классифицировать следующим образом:

а) $\mu_{k} \in \Lambda_{m_{k}}$ при некоторых $m_{k} \in\left\{0,1, \ldots, N_{R}\right\}$, и множество этих точек для каждого $k$ изоморфно $S^{1}$;

б) $\bar{P}_{S+1}=P_{S+2}$, или оба корня $\mu_{S+1}$ и $\mu_{S+2}$ лежат в одном и том же интервале $\Lambda_{m_{S}}$ при некоторых $m_{S}$, и множество этих точек определяется как $V=\left\{(P, \bar{P}) \in \mathcal{R}^{(2)}=\mathcal{R}^{2} / S_{2}\right\}$.

Случай "б" получается при рассмотрении двух точек $P, \bar{P} \in \mathcal{R}$, которые можно непрерывно перемешать по $\mathcal{R}$ вплоть до того, как $P=\bar{P}$, где $\pi(P) \in \Lambda_{m_{S}}$, и далее перемешать две точки по $\Lambda_{m_{S}}$ независимо от вешественной петли. Множество точек в этом случае включает $p_{\infty}^{ \pm}$. Из леммы 3.4 видно, что решение, соответствующее случаю "а", за исключением $\mu_{k} \in \Lambda_{0} \cup \Lambda_{N_{R}}$, является периодическим циклом, а во всех остальных случаях решения имеют сингулярности

Теперь можно доказать предложение 2.2 (см. также [4]).

ДОКАЗАТЕЛЬСТВО ПРЕДЛОЖЕНИЯ 2.2. ОбозНачим $\mathcal{R}_{\mathbb{R}}=C_{0} \sqcup \cdots \sqcup C_{m}$ при $m=$ $N_{R}-1$, где каждое $C_{i}$ порождает множество, соответствуюшее случаю "а" в лемме 3.4 т.е. если $P \in C_{i}$, то $\pi(P) \in \Lambda_{i}$. Тогда из леммы 3.4 следует, что симметрическое произведение $\mathcal{R}_{\mathbb{R}}^{(g)}=\left(\mathcal{R}^{g} / S_{g}\right) \mathbb{R}$ покрывается множествами

$$
U\left(i_{1}, \ldots, i_{j}\right):=C_{i_{1}} \times \cdots \times C_{i_{j}} \times V^{k}, \quad j+2 k=g
$$

Число несвязных множеств можно теперь вычислить следующим образом. Пусть $\left(n_{1}, \ldots, n_{g}\right)$ - возрастающая последовательность чисел $n_{k} \in\{0,1, \ldots, m\}$, а число $m=$ $N_{R}-1$ таково, что если $S \in\{1, \ldots, g\}$ - номер первого отличного от нуля $n_{k}$, тогда

$$
0=n_{1}=\cdots=n_{S-1}<n_{S}<n_{S+1}<\cdots<n_{g} \leqslant m .
$$

Это позволяет связать число $n_{k}$ из последовательности со случаями “а" и "б" в лемме 3.4 следуюшим образом:

1) из $n_{k}=0$ следует, что $\mu_{k}$ или принадлежит $\Lambda_{0} \cup \Lambda_{M}$, или относится к случаю "б", т.е. $P_{k} \in C_{0}$ или $\left(P_{k}, P_{l}\right) \in V$ для некоторых $P_{l}=\bar{P}_{k}$;

2) из $n_{k} \neq 0$ следует, что $\mu_{k}$ принадлежит ограниченному интервалу $\Lambda_{n_{k}}=$ $\left[\lambda_{2 n_{k}}, \lambda_{2 n_{k}+1}\right]$.

Подсчитывая теперь число последовательностей $\left(n_{1}, \ldots, n_{g}\right)$ при $n_{k} \in\{0,1, \ldots, m\}$ для каждого случая, когда $\mathcal{R}_{\mathbb{R}}$ имеет $N_{R}=m+1$ связных компонент, получаем число связных компонент $\mathcal{R}_{\mathbb{R}}^{(g)}$ в виде

$$
n\left(\mathcal{R}_{\mathbb{R}}^{(g)}\right)=\sum_{j=0}^{m}\left(\begin{array}{c}
m \\
j
\end{array}\right)=2^{m} .
$$


С другой стороны, записывая антиголоморфную инволюцию в матричном виде на решетке, заданной матрицей периодов $\Omega=(I, B)$ из $(2.4)$, можно показать, что каждая связная компонента $\left(\mathbb{C}^{g} / \Gamma\right)_{\mathbb{R}} \cong \operatorname{Jac}(\mathcal{R})_{\mathbb{R}}$ диффеоморфна вешественному тору $\mathbb{R}^{g} / \mathbb{Z}^{g}=$ $\mathbb{T}^{g}$.

Замечая, что $n\left(\mathrm{Jac}_{\mathbb{R}}\right)=n\left(\mathcal{R}_{\mathbb{R}}^{(g)}\right)$, тем самым завершаем доказательство.

Отметим, что число $\left(\begin{array}{c}m \\ j\end{array}\right)$ дает полное число последовательностей, вид которых позволяет отождествить их с множеством $U\left(i_{1}, \ldots, i_{j}\right)$ следуюшим образом:

$$
(\overbrace{0, \ldots, 0}^{g-j=S-1}, \overbrace{n_{S}, \ldots, n_{g}}^{j}) \equiv \begin{cases}V^{k} \times C_{n_{S}} \times \cdots \times C_{n_{g}}, & S=2 k+1, \\ V^{k} \times C_{0} \times C_{n_{S}} \times \cdots \times C_{n_{g}}, & S=2 k+2,\end{cases}
$$

где $n_{S} \neq 0$. Таким образом, каждую связную компоненту можно охарактеризовать числом компонент $C_{n_{i}}$ с ненулевыми $n_{i}$.

3.2. Поток Тоды на якобиане. Найдем на римановой поверхности поток Тоды, определяемый образом динамики $\mu_{n}$ при $g$-кратном отображении Абеля-Якоби $v_{g}$ : $\mathcal{R}^{(g)} \rightarrow \operatorname{Jac}(\mathcal{R})_{\mathbb{R}}$. Сначала заметим, что временна́я эволюция $\phi_{n}^{(1)}$ удовлетворяет уравнению

$$
\frac{d \phi_{n}^{(1)}}{d t}+a_{n-1} \phi_{n-1}^{(1)}=a_{N-1} \phi_{-1}^{(1)} \phi_{n}^{(1)}+a_{N} \phi_{n}^{(2)} .
$$

Это можно показать, вычислив $n$-й элемент этого уравнения,

$$
\frac{d \phi^{(1)}}{d t}-A \phi^{(1)}=c_{1} \phi^{(1)}+c_{2} \phi^{(2)} .
$$

Динамику $\mu_{n}$ можно тогда получить с учетом (3.4) при $\lambda=\mu_{j}$ и $n=N+1$, т.е.

$$
\left.\frac{d}{d t}\left(-a_{N} \prod_{k=1}^{N-1}\left(\lambda-\mu_{k}\right)\right)\right|_{\lambda=\mu_{j}}+a_{N} \phi_{N}^{(1)}\left(\mu_{j}\right)=a_{N} \phi_{N+1}^{(2)}\left(\mu_{j}\right) .
$$

Замечая, что $\phi_{N+1}^{(2)}=1 / \phi_{N}^{(1)}$, и используя $(3.3)$, получаем

$$
\frac{1}{y\left(\mu_{j}\right)} \frac{d \mu_{j}}{d t}= \pm \frac{1}{\prod_{k \neq j}\left(\mu_{j}-\mu_{k}\right)}
$$

где $y(\lambda)=\sqrt{P(\lambda)^{2}-4}$. Используя формулу интерполящии Лагранжа

$$
\sum_{j=1}^{N-1} \frac{\mu_{j}^{n}}{\prod_{k \neq j}\left(\mu_{j}-\mu_{k}\right)}= \begin{cases}0, & n<N-2, \\ 1, & n=N-2,\end{cases}
$$

после интегрирования получаем

$$
\sum_{j=1}^{N-1} \int_{\mu_{*}}^{\mu_{j}} \frac{\lambda^{n} d \lambda}{y(\lambda)}= \begin{cases}\delta_{n}, & n<N-2 \\ \pm t+\delta_{g-1}, & n=N-2\end{cases}
$$

Эти уравнения можно записать также в терминах голоморфных дифференциалов $\omega_{k}$, нормированных условиями (2.3), и тогда мы приходим к хорошо известному результату (см., например, [6]). 
ПРЕДЛОЖЕНИЕ 3.2. Периодический поток Тодь можно выразить как линейный поток на якобиане $\operatorname{Jac}(\mathcal{R}) \cong \mathbb{C}^{g} / \Gamma$, связанном с римановой поверхностью $\mathcal{R}$ рода $g=N-1$, m.e.

$$
\sum_{j=1}^{g} \int_{\mu_{*}}^{\mu_{j}} \omega_{k}= \pm C_{k, g-1} t+\delta_{k}^{\prime} \quad(\bmod \Gamma), \quad k=1, \ldots, g
$$

где $\delta^{\prime}-$ константа, а $(g \times g)$-матрица $\left(C_{k, n}\right)$ определяется условием нормировки (2.3) прu

$$
\omega_{k}=\sum_{n=0}^{g-1} C_{k, n} \frac{\lambda^{n} d \lambda}{y}
$$

С использованием предложений 3.1 и 3.2 мы построили отображения

$$
\begin{gathered}
Z_{\mathbb{R}}^{P}(\gamma) \stackrel{\varphi}{\longrightarrow} \mathcal{R}_{\mathbb{R}}^{(g)} \stackrel{v_{g}}{\longrightarrow} \operatorname{Jac}(\mathcal{R})_{\mathbb{R}} \\
\left(b_{1}, \ldots, b_{N}\right) \longmapsto\left[\mu_{1}, \ldots, \mu_{g}\right] \longmapsto\left[\sum_{j=1}^{g} \int_{\mu_{*}}^{\mu_{j}} \omega\right],
\end{gathered}
$$

где $\omega=\left(\omega_{1}, \ldots, \omega_{g}\right)$ и $g$-кратное отображение Абеля-Якоби $v_{g}$ является сюръективным [5]. Поскольку прообраз каждой связной компоненты в $\operatorname{Jac}(\mathcal{R})_{\mathbb{R}}$ задается в $\mathcal{R}_{\mathbb{R}}^{(g)}$ выражением (3.9), обозначим

$$
\left(\mathbb{T}^{g}\right)_{j} \cong\left(0, \ldots, 0, n_{g-j+1}, \ldots, n_{g}\right), \quad 0 \leqslant j \leqslant m \leqslant g
$$

и тогда

$$
\operatorname{Jac}(\mathcal{R})_{\mathbb{R}} \cong \bigsqcup_{j=0}^{m} \mathcal{M}_{j}, \quad \mathcal{M}_{j}=\overbrace{\left(\mathbb{T}^{g}\right)_{j} \sqcup \cdots \sqcup\left(\mathbb{T}^{g}\right)_{j}}^{\left(\begin{array}{c}
m \\
j
\end{array}\right)} .
$$

ЗАмЕЧАнИЕ 3.1. Поскольку тор $\left(\mathbb{T}^{g}\right)_{j}$ является вешественной частью комплексной абелевой группы $\mathrm{Jac}(\mathcal{R}) \cong \mathbb{C}^{g} / \Gamma$, то он представляет собой вешественную абелеву группу, диффеоморфную $g$-мерному вешественному тору $\mathbb{R}^{g} / \mathbb{Z}^{g}$. Введем множество

$$
\left(\mathbb{T}^{k}\right)_{j} \cong(\overbrace{0, \ldots, 0, n_{g-j+1}, \ldots, n_{g}}^{k}), \quad j \leqslant k<g .
$$

Однако, если $k \neq j$, оно может не оказаться $k$-мерным вешественным тором. В настоящей работе мы не будем обсуждать детали определения $\left(\mathbb{T}^{k}\right)_{j}$, отметим только, что его топология определяется множеством $V=\left\{(P, \bar{P}) \in \mathcal{R}^{(2)}\right\}$ (см. [7]). Подробнее это исследование будет опубликовано отдельно. 
ЗАмечАниЕ 3.2. Уравнение (3.10) можно выразить в виде интегрируемой системы Мамфорда (3.7) (см. [7])

$$
\frac{d M_{g}(\lambda)}{d t}=\left[M_{g}(\lambda), B_{g}(\lambda)\right]
$$

где матрицы $M_{g}(\lambda)$ и $B_{g}(\lambda)$ даются тройками системы Мамфорда $\left(u_{g}, v_{g}, w_{g}\right)$ :

$$
M_{g}(\lambda)=\left(\begin{array}{cc}
v_{g}(\lambda) & u_{g}(\lambda) \\
w_{g}(\lambda) & -v_{g}(\lambda)
\end{array}\right), \quad B_{g}(\lambda)=\frac{1}{2}\left(\begin{array}{cc}
0 & 1 \\
b_{g}(\lambda) & 0
\end{array}\right)
$$

Здесь $b_{g}(\lambda)$ - приведенный полином степени $g+2$, который единственным образом определяется из согласованности системы (3.16). Уравнение можно обобщить для описания динамики на дивизоре (см. ниже).

3.3. Тета-функция Римана и тета-дивизор. Здесь мы приведем решение периодической решетки Тоды в терминах тета-функции Римана, а также обсудим геометрию множества сингулярных точек, определяемых тета-дивизором нулей тета-функции Римана.

Сначала определим верхнюю полуплоскость Зигеля $\mathfrak{S}_{g}$ как множество симметричных комплексных $(g \times g)$-матриц с положительно определенной мнимой частью, т.е.

$$
\mathfrak{S}_{g}:=\left\{B \in \mathfrak{M}(g, \mathbb{C}): B^{\mathrm{t}}=B, \operatorname{Im} B>0\right\} .
$$

Тогда на произведении $\mathbb{C}^{g} \times \mathfrak{S}_{g}$ можно определить тета-функцию Римана

$$
\vartheta(z \mid B)=\sum_{m \in \mathbb{Z}^{g}} \mathbf{e}\left(m^{\mathrm{t}} z+\frac{1}{2} m^{\mathrm{t}} B m\right)
$$

где $\mathbf{e}(x)=e^{2 \pi \sqrt{-1} x}$. Поскольку $\operatorname{Im} B>0$, приведенный выше ряд сходится равномерно на компактных множествах на $\mathbb{C}^{g} \times \mathfrak{S}_{g}$ к голоморфной функции. Легко видеть, что тета-функция удовлетворяет соотношениям квазипериодичности

$$
\begin{aligned}
& \vartheta\left(z+E_{k} \mid B\right)=\vartheta(z \mid B), \\
& \vartheta\left(z+B_{k} \mid B\right)=\mathbf{e}\left(-z_{k}-B_{k, k}\right) \vartheta(z \mid B),
\end{aligned}
$$

где $E_{1}, \ldots, E_{g}, B_{1}, \ldots, B_{g}-(2 g)$-векторы-столбцы из матрицы периодов $\Omega=(I, B)$. Как следует из этих уравнений, для каждой фиксированной матрицы $B \in \mathfrak{S}_{g}$ дивизор тета-функции Римана инвариантен относительно трансляций на элементы решетки $\Gamma$, порожденной $E_{1}, \ldots, B_{g}$, и он индуцирует дивизор $\Theta_{0}$ на $\operatorname{Jac}(\mathcal{R})$, который называется тета-дивизором,

$$
\Theta_{0}:=\{[z] \in \operatorname{Jac}(\mathcal{R}): \vartheta(z \mid B)=0\}
$$


Рассмотрим функцию $f: \mathcal{R} \rightarrow \mathbb{C}$, определяемую соотношением

$$
f(P):=\vartheta(\tilde{v}(P)-c \mid B),
$$

где $c \in \mathbb{C}^{g}$ - произвольный постоянный вектор, а $\tilde{v}$ - подъем отображения Абеля-Якоби $v$, т.е.

$$
\tilde{v}: \mathcal{R}^{\prime} \longrightarrow \mathbb{C}^{g}, \quad P \longmapsto\left(\int_{P_{0}}^{P} \omega\right),
$$

где $\mathcal{R}^{\prime}$ - многоугольник с $4 g$ сторонами, полученный путем разрезания $\mathcal{R}$ по базисным циклам, $\left\{\left(\alpha_{k}, \beta_{k}\right): k=1, \ldots, g\right\}, \mathrm{a} \omega=\left(\omega_{1}, \ldots, \omega_{g}\right)$ - голоморфный дифференциал. Тогда стандартной является следуюшая лемма (доказательство см., например, в [8]).

ЛЕмма 3.5. Для нулей функиии $f(P)$ выполнены следующие условия:

а) $f(P)$ имеет $g$ нулей на $\mathcal{R}$ (считаемых с учетом их кратностей);

б) Если $P_{1}, \ldots, P_{g}-$ нули функиии $f(P)$, то

$$
\sum_{j=1}^{g} u\left(P_{j}\right)+[\kappa]=[c]
$$

где к-константа Римана.

Отсюда следует

ПРЕДЛОЖЕНИЕ 3.3. Пусть $Q_{1}, \ldots, Q_{g}$ - множество произвольнъц точек на $\mathcal{R}$. Тогда функиия $\vartheta\left(\tilde{v}(P)-\sum_{j=1}^{g} \tilde{v}\left(Q_{j}\right)-\kappa \mid B\right)$ имеет $g$ нулей точно в $P=$ $Q_{1}, \ldots, Q_{g}$.

С геометрической точки зрения предложение 3.3 представляет хорошо известный факт о тета-дивизоре $\Theta_{0}$ :

СлЕДСТвИЕ. Тета-дивизор $\Theta_{0}$ на якобиане $\operatorname{Jac}(\mathcal{R})$ удовлетворяет соотношению

$$
\Theta_{0}=W^{g-1}+[\kappa]
$$

где $W^{g-1}$ - образ г-кратного отображсения Абеля-Якоби $v_{r}$ при $r=g-1$, т.е.

$$
\begin{aligned}
v_{r}: \mathcal{R}^{(r)} & \longrightarrow \operatorname{Jac}(\mathcal{R}) \\
{\left[Q_{1}, \ldots, Q_{r}\right] } & \longmapsto\left[\sum_{j=1}^{r} \int_{Q_{0}}^{Q_{j}} \omega\right]
\end{aligned}
$$


ПримеР 3.1. Риманова поверхность рода $g=2$ и дивизор $\Theta_{0}$. В случае компактной римановой поверхности $\mathcal{R}$ рода $g=2$ образ $W^{1}$ изоморфен римановой поверхности, и, следовательно, якобиан $\operatorname{Jac}(\mathcal{R})$ содержит риманову поверхность рода $g=2$ как замкнутое подмногообразие $\Theta_{0} \cong \mathcal{R}$. Таким образом, имеем $\operatorname{Jac}(\mathcal{R})_{\mathbb{R}} \cap \Theta_{0} \cong \mathcal{R}_{\mathbb{R}}$.

Теперь можно найти решение периодической решетки Тоды, решая задачу обрашения Якоби для $\mu_{j}$ из уравнения (3.2). Решение $\mu_{j}(t)=\pi\left(P_{j}\right)$ дается нулем тета-функции

$$
f(P)=\vartheta(\tilde{v}(P)-c \mid B)=0, \quad c= \pm C_{g-1} t+\kappa^{\prime},
$$

где $\kappa^{\prime}=\kappa+\delta^{\prime}$, а $C_{g-1}=\left(C_{1, g-1}, \ldots, C_{g, g-1}\right)$ - вектор, представляющий собой столбец матрицы $\left(C_{k, n}\right)$ из (3.12). Из формулы (3.6) следует, что для получения решения $b_{k}(t)$ нам требуется именно сумма корней $\mu_{n}$. Сумму $\sum_{j=1}^{g} \mu_{j}$ можно получить путем интегрирования, т.е.

$$
\frac{1}{2 \pi \sqrt{-1}} \int_{\partial \mathcal{R}^{\prime}} \pi(P) d \ln f(P)=\sum_{j=1}^{g} \mu_{j}+\pi\left(p_{\infty}^{+}\right)+\pi\left(p_{\infty}^{-}\right)
$$

где $\pi\left(p_{\infty}^{ \pm}\right)$- вклады от бесконечности. Окончательно получаем (подробности доказательства см. в работе [6])

ПРеДЛОЖенИЕ 3.4. Решение $\left(a_{1}, \ldots, a_{N}, b_{1}, \ldots, b_{N}\right)$ решетки Тодъ дается въражсением

$$
\begin{aligned}
& a_{k}(t)=\frac{\tau_{k+1}(t) \tau_{k-1}(t)}{\left(\tau_{k}(t)\right)^{2}}, \\
& b_{k}(t)=\frac{d}{d t} \ln \frac{\tau_{k}(t)}{\tau_{k-1}(t)},
\end{aligned}
$$

əде $\tau_{k}(t)=\vartheta\left(c^{\prime} t+c k+\delta_{0} \mid B\right) n p u c^{\prime}= \pm C_{g-1}, \quad c=\tilde{v}\left(p_{\infty}^{-}\right)-\tilde{v}\left(p_{\infty}^{+}\right) u \delta_{0}=\tilde{v}\left(p_{\infty}^{+}\right)-$ $\kappa-\delta^{\prime}$.

Теперь ясно, что если $\tau_{k}(t)$ обращается в нуль при некоторых $k$ и $t$, то функции $a_{k}(t)$ и $b_{k \pm 1}(t)$ становятся сингулярными. Таким образом, дивизор $\Theta$ дается суммой множеств нулей тау-функций, которые являются тета-дивизором $\Theta_{0}$ и его трансляциями $\Theta_{k}$, т.е.

$$
\Theta=\bigcup_{k=0}^{g} \Theta_{k}, \quad \Theta_{k}=\Theta_{0}+k\left(\tilde{v}\left(p_{\infty}^{-}\right)-\tilde{v}\left(p_{\infty}^{+}\right)\right) .
$$

Таким образом, изоспектральное множество $Z_{\mathbb{R}}^{P}(\gamma)$ можно компактифицировать путем склейки по тета-дивизорам. Можно также заметить, что кратность нуля функции $\tau_{k}(t)$ дает кратность пересечения дивизоров. Комплексный вариант геометрии дивизоров был разработан в статье [2] (см. также [9] для случая конечных непериодических решеток Тоды). Настояшая работа посвяшена вешественному варианту такого исследования, и наша основная цель заключается в том, чтобы исследовать структуру склейки в рамках теории Ли в терминах представления аффинной группы Вейля $W$. 
ЗАмечАниЕ 3.3. Для решения вида (3.19) легко показать, что тау-функции $\tau_{k}(t)$ удовлетворяют билинейным соотношениям Хироты

$$
\tau_{k} \tau_{k}^{\prime \prime}-\left(\tau_{k}^{\prime}\right)^{2}=\tau_{k+1} \tau_{k-1}
$$

где $\tau_{k}^{\prime}=d \tau_{k} / d t$ и $\tau_{k}^{\prime \prime}=d^{2} \tau_{k} / d t^{2}$. Эта формула будет полезна при исследовании вырождения нулей, которое характеризует пересечение дивизоров.

\section{4. ГИПОТЕЗЫ О ТОПОЛОГИИ $\operatorname{Jac}(\mathcal{R})_{\mathbb{R}}$}

Здесь мы выскажем несколько гипотез о геометрии дивизора на $\operatorname{Jac}(\mathcal{R})_{\mathbb{R}}$ и топологии афффинной части $\operatorname{Jac}(\mathcal{R})_{\mathbb{R}}$.

4.1. Геометрия дивизора $\Theta$. Напомним, что $\Theta_{k}$ является трансляцией тета-дивизора $\Theta_{0}$ на $k(Q-P)$, где $Q=v\left(p_{\infty}^{-}\right), P=v\left(p_{\infty}^{+}\right)$, так что $\Theta_{k} \cong \Theta_{0} \cong W^{g-1}$. Тогда, следуя работе [2], определим множества $\Theta_{J}$ для каждого подмножества $J \varsubsetneqq\{0,1, \ldots, g\}$ как пересечения с $\Theta_{k}$ :

$$
\Theta_{J}:=\bigcap_{k \in J} \Theta_{k}, \quad \operatorname{dim}_{\mathbb{C}} \Theta_{J}=g-|J|
$$

Отметим, что $\Theta_{J} \supset \Theta_{J^{\prime}}$, если $J \subset J^{\prime}$, и $\Theta_{J} \cong \mathcal{R}$, если $|J|=g-1$. Тогда мы имеем стратификацию якобиана:

$$
\operatorname{Jac}(\mathcal{R}) \supset \Theta^{(1)} \supset \Theta^{(2)} \supset \cdots \supset \Theta^{(g)}, \quad \Theta^{(k)}:=\bigcup_{|J|=k} \Theta_{J} .
$$

Мы также имеем клеточное разложение дивизора $\Theta$ в терминах этих множеств:

$$
\Theta=\bigsqcup_{J \varsubsetneqq\{0,1, \ldots, g\}} D_{J}, \quad D_{J}:=\Theta_{J} \bigcap_{k \notin J} \Theta_{k}^{c}
$$

где $\Theta_{k}^{c}$ - дополнение к $\Theta_{k}$ в $\mathrm{Jac}(\mathcal{R})$. Для того чтобы вычислить пересечения $\operatorname{Jac}(\mathcal{R})_{\mathbb{R}} \cap$ $\Theta_{J}$, сформулируем следуюшую лемму.

Лемма 4.1. Пусть $\left(\mathbb{T}^{g}\right)_{j}-g$-мерный тор, определяемый уравнением (3.14). Тогда имеем

$$
\left(\mathbb{T}^{g}\right)_{j} \cap \Theta_{J}= \begin{cases}\left(\mathbb{T}^{g-|J|}\right)_{j}, & |J| \leqslant g-j \\ \varnothing, & |J|>g-j .\end{cases}
$$


ДокАЗАТЕЛЬСтво. Сначала рассмотрим случай, когда $J=\{k\}$ при $k=0,1, \ldots, g$. Поскольку имеется изоморфизм между $\Theta_{k}$ и образом $(g-1)$-кратного симметрического произведения римановых поверхностей, $\Theta_{k} \cong W^{g-1}$ (предложение 3.1 ), то множество $\Theta_{k} \cap\left(\mathbb{T}^{g}\right)_{j}$ состоит из элементов, обозначаемых как $(\overbrace{0, \ldots, 0}^{g-j-1}, n_{g-j+1}, \ldots, n_{g})$, т.е. один из первых $(g-j)$ элементов в $\left(\mathbb{T}^{g}\right)_{j}$ фиксирован в точке бесконечности. Отсюда следует утверждение леммы. Нетрудно видеть, что при $|J|>1$ доказательство аналогично.

ЗАмЕчАниЕ 4.1. Лемма 4.1 является следствием формулы Пуанкаре

$$
\Theta_{J} \cong|J| ! W^{g-|J|} \quad \text { (гомологично), }
$$

а многообразие $\left(\mathbb{T}^{g-|J|}\right)_{j}$ в обшем случае сингулярно. Однако представляется, что $\left(\mathbb{T}^{g-|J|}\right)_{j}$ является гладким, если $J$ состоит из последовательных чисел $J=\{S+1, \ldots$ $\ldots, S+|J|\}(\bmod N)($ см. теорему 5 и следствие 1 в работе [2]).

Поскольку дивизор $\Theta$ является суммой нулей тау-функций $\tau_{k}(t)=\vartheta(k(Q-P)+$ $R(t) \mid B)$, клетка $D_{J}$ также определяется как

$$
R\left(t_{J}\right) \in D_{J} \cap \operatorname{Jac}(\mathcal{R})_{\mathbb{R}} \stackrel{\text { def }}{\Longleftrightarrow} \tau_{k}\left(t_{J}\right)=0 \quad \text { тогда и только тогда, когда } \quad k \in J .
$$

На $D_{J}$ можно определить кратность $m_{k}$ нуля функции $\tau_{k}(t)$ при $k \in J$, которая описывает геометрию пересечения дивизоров $\Theta_{k}$ при $k \in J$.

ЛЕмма 4.2. Пусть $J$ - множество последовательных чисел, задаваемое как $\{i+1, \ldots, i+S\}(\bmod N)$. Тогда вблизи своего нуля $t=t_{J}$ функция $\tau_{k}(t)$ имеет вид

$$
\tau_{i+k}(t) \simeq\left(t-t_{J}\right)^{m_{k}}+\cdots, \quad m_{k}=k(S+1-k), \quad 1 \leqslant k \leqslant S
$$

ДоКАЗАТЕЛЬСТво. Подставляя (4.3) в (3.21) и используя $\tau_{i}\left(t_{J}\right) \neq 0$, имеем $m_{k}=$ $k\left(m_{1}+1-k\right)$. Тогда $\tau_{i+S+1}\left(t_{J}\right) \neq 0$, откуда следует, что $m_{1}=S$ (см. также [1]).

Как показано в работе [10], дивизор $D_{J}$ можно параметризовать с помошью так называемой предельной матрицы $L_{J}$, определяемой как

$$
L_{J}:=\lim _{t \rightarrow t_{J}} n_{J}^{-1}(t) L(t) n_{J}(t) \quad \text { при некоторых } \quad n_{J}(t) \in N_{-},
$$

где $L_{J}$ имеет $2 N-|J|$ переменных, а $N_{-}$- множество нижнетреугольных матриц с единицами на диагоналях. Матрица $n_{J}$ единственным образом определяется структурой сингулярностей, даваемой леммой 4.2. Для множества $J=\left\{i_{1}, \ldots, i_{n}\right\}$ предельную матрицу $L_{J}$ можно построить из цепочки матриц, начиная с исходной матрищы $L_{P}$ :

$$
L_{P} \rightarrow L_{J_{1}} \rightarrow L_{J_{2}} \rightarrow \cdots \rightarrow L_{J}
$$


где $J_{k}=\left\{i_{1}, \ldots, i_{k}\right\}$ при $k<n=|J|$ и $J_{n}=J$. Матрицу $n_{J_{k}} \in N_{-}$легко найти в терминах $L_{J_{k}}$, откуда мы получаем предел $n_{J_{k}}^{-1} L_{J_{k}} n_{J_{k}} \rightarrow L_{J_{k+1}}$. Тогда $L_{J_{k}}$ параметризует дивизор $D_{J_{k}}$, причем $\operatorname{codim}_{\mathbb{C}} D_{J_{k}}=k$ (деталям этого исследования будет посвящена отдельная работа).

Теперь с помошью предельной матрицы $L_{J}$ можно определить интегрируемую систему на $D_{J}$ в терминах троек системы Мамфорда $\left(u_{d}(\lambda), v_{d}(\lambda), w_{d}(\lambda)\right)$ при $d=\operatorname{dim}_{\mathbb{C}} D_{J}=$ $g-|J|[5],[7]$. Здесь тройки удовлетворяют соотношениям

$$
y^{2}=P_{J}(\lambda)^{2}-4=u_{d} w_{d}+v_{d}^{2}, \quad \operatorname{det}\left(L_{J}-\lambda I\right)=-\left(z+z^{-1}-P_{J}(\lambda)\right),
$$

где $u_{d}(\lambda)$ - приведенный полином степени $d, v_{d}(\lambda)$ - полином степени $d-1$ при $v_{d}\left(\mu_{j}\right)=$ $y\left(\mu_{j}\right)$ во всех случаях, когда $u_{d}\left(\mu_{j}\right)=0$, а $w_{d}(\lambda)$ - приведенньй полином степени $2 g+$ $2-d$. Нули $u_{d}(\lambda)$ параметризуют многообразие $W^{d} \subset \operatorname{Jac}(\mathcal{R})$ - образ симметрических произведений $\mathcal{R}^{(d)}$ при $k$-кратном отображении Абеля-Якоби (это утверждение в терминах дивизора $\operatorname{Div}_{0}^{+, d}(\mathcal{R})$ см. в [5]). В частности, если $J$ дается множеством $(g-d)$ последовательных чисел, а именно $J=\{1, \ldots, g-d\}$, то $u_{d}(\lambda)$ можно выразить как

$$
u_{d}(\lambda)=(-1)^{d} \Delta_{N-d+1, N}(\lambda)=\lambda^{d}-\left(\sum_{i=N-d+1}^{N} b_{i}\right) \lambda^{d-1}+\cdots=\prod_{i=1}^{d}\left(\lambda-\mu_{i}\right),
$$

где $\Delta_{m, n}(\lambda)$ дается уравнением (1.9) (см. ниже пример 4.1). Тогда интегрируемая система на $D_{J}$ имеет вид (3.16), откуда следует (3.10) с индексом $j$, принимаюшим значения $1 \leqslant j \leqslant d=g-|J|$. Эту систему можно использовать для демонстрации гладкости $\Theta_{J}$ для таких $J$. Отметим, что задача обрашения Якоби для такой системы является неполной, и многообразие решений не может быть дополнено до абелева многообразия (в этом смысле система не является алгебраически интегрируемой) [11]. Исследованию деталей решения таких интегрируемых систем будет посвящена отдельная работа.

ПримеР 4.1. $D_{J} \subset \mathrm{Jac}(\mathcal{R})$ для римановой поверхности рода $g=3$.

1. $J=\{1,2\}$. Предельная матрица $L_{\{1,2\}}$ имеет вид

$$
L_{\{1,2\}}=\left(\begin{array}{cccc}
0 & 1 & 0 & 0 \\
0 & 0 & 1 & 0 \\
\xi_{3} & -\xi_{2} & \xi_{1} & 1-\zeta_{1} z^{-1} \\
z-\zeta_{2} & 0 & 0 & \mu_{1}
\end{array}\right)
$$

где $(3 \times 3)$-подматрица в верхнем левом углу является сопровождаюшей матрищей для соответствуюшей подматрицы, т.е. $\xi_{1}=b_{1}+b_{2}+b_{3}, \xi_{2}=b_{1} b_{2}+b_{2} b_{3}+b_{3} b_{1}-a_{1}-a_{2}, \xi_{3}=$ $b_{1} b_{2} b_{3}-a_{1} b_{3}-a_{2} b_{1}$ и $\zeta_{1}=-a_{1} a_{3}, \zeta_{2}=-a_{2} a_{4}, \mu_{1}=b_{4}$. Определяемая матрицей $L_{\{1,2\}}$ спектральная кривая дается уравнением

$$
\operatorname{det}\left(L_{\{1,2\}}-\lambda I\right)=-\left(z+I_{5} z^{-1}-P_{\{1,2\}}(\lambda)\right), \quad P_{\{1,2\}}(\lambda)=\lambda^{4}+\sum_{k=1}^{4}(-1)^{k} I_{k} \lambda^{4-k} .
$$


Здесь инварианты $I_{1}, \ldots, I_{5}$ задаются формулами

$$
\begin{array}{ll}
I_{1}=\xi_{1}+\mu_{1}=0, & I_{2}=\xi_{1} \mu_{1}+\xi_{2}, \quad I_{3}=\xi_{2} \mu_{1}+\xi_{3}, \\
I_{4}=\xi_{3} \mu_{1}+\zeta_{1}+\zeta_{2}, & I_{5}=\zeta_{1} \zeta_{2}=1 .
\end{array}
$$

Полиномы $u_{1}(\lambda), v_{1}(\lambda)$ троек системы Мамфорда выражаются в виде $u_{1}(\lambda)=\lambda-\mu_{1}$, $v_{1}(\lambda)= \pm y\left(\mu_{1}\right)$. Тогда изоспектральное многообразиев $\operatorname{Jac}(\mathcal{R})_{\mathbb{R}}$ со связями $\tau_{1}=\tau_{2}=0$ имеет вид

$$
\begin{aligned}
D_{\{1,2\}} \cap \operatorname{Jac}(\mathcal{R})_{\mathbb{R}} & =\left\{\left(\xi_{1}, \xi_{2}, \xi_{3}, \zeta_{1}, \zeta_{2}, \mu_{1}\right) \in \mathbb{R}^{6}: I_{k}=\gamma_{k} \in \mathbb{R}, k=1, \ldots, 5\right\} \cong \\
& \cong\left\{\left(\zeta_{1}, \mu_{1}\right) \in \mathbb{R}^{2}: \zeta_{1}+\zeta_{1}^{-1}-P_{\{1,2\}}\left(\mu_{1}\right)=0\right\} \cong \mathcal{R}_{\mathbb{R}} \cap \mathcal{R}_{0} .
\end{aligned}
$$

Интегрируемая система $d u_{1}(\lambda) / d t=v_{1}(\lambda)$ записывается на этом многообразии как

$$
\frac{d \mu_{1}}{d t}= \pm y\left(\mu_{1}\right)= \pm \sqrt{P_{\{1,2\}}\left(\mu_{1}\right)^{2}-4}
$$

и описывает динамику на кривой $\mathcal{R}_{\mathbb{R}}$.

2. $J=\{1,3\}$. Предельная матрица в этом случае имеет вид

$$
L_{\{1,3\}}=\left(\begin{array}{cccc}
0 & 1 & 0 & 0 \\
-\xi_{2} & \xi_{1} & 1-\zeta_{1} z^{-1} & 0 \\
0 & 0 & 0 & 1 \\
z-\zeta_{2} & 0 & -\eta_{2} & \eta_{1}
\end{array}\right),
$$

где $\xi_{1}=b_{1}+b_{2}, \xi_{2}=b_{1} b_{2}-a_{1}, \eta_{1}=b_{3}+b_{4}, \eta_{2}=b_{3} b_{4}-a_{3}, \zeta_{1}=a_{4} b_{1} b_{2}$ и $\zeta_{2}=a_{2} b_{1} b_{3}$. Инварианты $I_{1}, \ldots, I_{5}$ задаются следуюшим образом:

$$
\begin{gathered}
I_{1}=\xi_{1}+\eta_{1}=0, \quad I_{2}=\xi_{1} \eta_{1}+\xi_{2}+\eta_{2}, \quad I_{3}=\xi_{1} \eta_{2}+\xi_{2} \eta_{1}, \\
I_{4}=\xi_{2} \eta_{2}+\zeta_{1}+\zeta_{2}, \quad I_{5}=\zeta_{1} \zeta_{2}=1,
\end{gathered}
$$

откуда мы имеем изоспектральное многообразие со связями $\tau_{1}=\tau_{3}=0$

$$
\begin{aligned}
D_{\{1,3\}} \cap \operatorname{Jac}(\mathcal{R})_{\mathbb{R}} & =\left\{\left(\xi_{1}, \xi_{2}, \eta_{1}, \eta_{2}, \zeta_{1}, \zeta_{2}\right) \in \mathbb{R}^{6}: I_{k}=\gamma_{k} \in \mathbb{R}, k=1, \ldots, 5\right\} \cong \\
& \cong\left\{\left(\zeta_{1}, \xi_{1}\right) \in \mathbb{R}^{2}: \zeta_{1}+\zeta_{1}^{-1}+Q\left(\xi_{1}\right)=0\right\},
\end{aligned}
$$

где $Q\left(\xi_{1}\right)$ - рациональная функция $\xi_{1}$, задаваемая как

$$
Q\left(\xi_{1}\right)=\frac{1}{4}\left(\xi_{1}^{2}+I_{2}\right)^{2}-\frac{I_{3}^{2}}{4 \xi_{1}^{2}}-I_{4} .
$$

Тогда соответствующий дивизор $\Theta_{\{1,3\}}$ в $\operatorname{Jac}(\mathcal{R})_{\mathbb{R}}$ содержит одномерное несвязное сингулярное многообразие, состоящее из двух окружностей, трансверсально пересекающихся в двух различных точках бесконечности. Таким образом, дивизоры $\Theta_{J}$ имеют весьма различную топологическую структуру в зависимости от множества $J$, и эти различия можно охарактеризовать с помощью поддиаграмм Дьнкина, связанных с $\tau_{k}=0$ при $k \in J$ (см. [2], [9], деталям исследования будет посвящена отдельная работа). 
4.2. Аффинная часть якобиана. Сначала сформулируем следующую гипотезу, касающуюся структуры аффинных частей якобиана $\operatorname{Jac}(\mathcal{R})_{\mathbb{R}}$, изоморфного $Z_{\mathbb{R}}^{P}(\gamma)$ (см. (2.6) для случая, когда $g=1$ ).

ГипотеЗА 4.1. Аффинная часть $\operatorname{Jac}(\mathcal{R})_{\mathbb{R}}$ имеет разложение

$$
\operatorname{Jac}(\mathcal{R})_{\mathbb{R}} \backslash \Theta \cong \bigsqcup_{j=0}^{m} \mathcal{M}_{j}^{\circ}, \quad m=N_{R}-1 \leqslant g
$$

причем $\mathcal{M}_{j}^{\circ}$ состоит из $\left(\begin{array}{c}m \\ j\end{array}\right)$ несвязных компонент $\left(\mathbb{T}^{g}\right)_{j} \backslash \Theta$, где каждый тор $\left(\mathbb{T}^{g}\right)_{j}$ разбивается на $g+1$ иилиндров $\mathbb{R}^{g-j} \times \mathbb{T}^{j}$ по дивизорам $\Theta_{k}$ из (3.20),

$$
\left(\mathbb{T}^{g}\right)_{j} \backslash \Theta=\left(\mathbb{R}^{g-j} \times \mathbb{T}^{j}\right) \sqcup \cdots \sqcup\left(\mathbb{R}^{g-j} \times \mathbb{T}^{j}\right)
$$

Мотивировка этой гипотезы такова. Напомним, что $\left(\mathbb{T}^{g}\right)_{j}$ занумерованы с помощью $(\overbrace{0, \ldots, 0, n_{g-j+1}, \ldots, n_{g}}^{g})$, где каждый элемент с меткой 0 содержит дивизоры, соответствующие $p_{\infty}^{ \pm} \in \mathcal{R}$. Удаляя эти точки через $g+1$ дивизоров $\Theta_{k}, \Theta=\bigcup_{k=0}^{g} \Theta_{k}$, мы рассчитываем получить $g+1$ несвязных цилиндров. Чтобы доказать гипотезу 4.1 , надо определить структуру пересечений дивизоров на $\left(\mathbb{T}^{g}\right)_{j}$.

Рассмотрим правило склейки таких несвязных цилиндров в $\left(\mathbb{T}^{g}\right)_{j} \backslash \Theta$, которые отмечены набором знаков $\left(\epsilon_{1}, \ldots, \epsilon_{N}\right)$, где $\epsilon_{k}=\operatorname{sign} a_{k}$. Пусть $\Sigma$ - набор периодических последовательностей знаков:

$$
\Sigma(N):=\left\{\left(\ldots, \sigma_{k-1}, \sigma_{k}, \sigma_{k+1}, \ldots\right): \sigma_{k} \in\{ \pm\}, \sigma_{k+N}=\sigma_{k} \forall k \in \mathbb{Z}\right\}
$$

Обозначим также через $\sigma^{0} \in \Sigma(N)$ специальный элемент, состоящий только из знаков + . Далее занумеруем каждьй цилиндр $\mathbb{R}^{g-j} \times \mathbb{T}^{j}$ периодической последовательностью знаков $\sigma$ и свяжем знак $\epsilon_{k}=\operatorname{sign} a_{k}$ со знаком $\epsilon_{k}=\sigma_{k} \sigma_{k+1}$.

Определим далее перестановку $S_{k}$ на $\Sigma(N)$,

$$
\begin{aligned}
S_{k}: \Sigma(N) & \longrightarrow \Sigma(N), \\
\left(\ldots, \sigma_{k}, \sigma_{k+1}, \ldots\right) & \longmapsto\left(\ldots, \sigma_{k+1}, \sigma_{k}, \ldots\right),
\end{aligned}
$$

где действие $S_{k}$ периодично с периодом $N$, т.е. $k \in \mathbb{Z} / N \mathbb{Z}$. Множество всех перестановок есть аффинная группа Вейля $W$, связанная с аффинной алгеброй Каца-Муди $A_{N-1}^{(1)}$ для $\mathfrak{s l}(N)$ :

$$
W:=\left\langle\begin{array}{cc}
S_{1}, \ldots, S_{N}: & S_{k}^{2}=e, \quad\left(s_{k} S_{k+1}\right)^{3}=e(\bmod N) \\
\left(S_{k} S_{j}\right)^{2}=e, \quad k \neq j, j \pm 1(\bmod N)
\end{array}\right\rangle
$$

где $e$ - единица. Тогда можно показать, что действие $W$ на знаках обладает следующим свойством периодичности. 
ЛЕмма 4.3. Для всякого $\sigma \in \Sigma(N) \backslash\left\{\sigma^{0}\right\}$ существует периодическое действие длинь $N, w_{N}^{(\sigma)}:=S_{i_{N}} \ldots S_{i_{1}} \in W$ nри $i_{j} \neq i_{k}$, если $j \neq k$, такое, что:

1) $w_{N}^{\sigma} \sigma=\sigma$

2) $S_{i_{j}} \ldots S_{i_{1}} \sigma \neq S_{i_{k}} \ldots S_{i_{1}} \sigma$ npu $0 \leqslant j<k<N$;

3) $N$ является минимальным числом, т.е. если имеется $w(\neq e) \in W$ такое, что $w \sigma=\sigma$, то $l(w) \geqslant N$, где $l(w)-$ длина әлемента $w \in W$.

\section{ДоказАтельство. Рассмотрим последовательность $\quad(\overbrace{+\cdots+}^{n_{1}} \overbrace{-\cdots-}^{n_{2}} \overbrace{+\cdots+}^{n_{3}}$} $\overbrace{-\cdots-}^{n_{4}})$ при $\sum_{k=1}^{4} n_{k}=N$ и предположим, что $\sigma$ дается ее периодическим расширением периода $N$. Сначала переместим знак + из $n_{1}$-й позиции в $\left(n_{1}+n_{2}\right)$-ю позицию, применяя перестановку $w_{1}=S_{n_{1}+n_{2}-1} \ldots S_{n_{1}+1} S_{n_{1}}$ при $l\left(w_{1}\right)=n_{2}$. Переместим также знак + из $\left(n_{1}+n_{2}+n_{3}\right)$-й позиции в $N$-ю позицию с помощью перестановки $w_{2}=S_{N-1} \ldots S_{n_{1}+n_{2}+n_{3}}$ при $l\left(w_{2}\right)=n_{4}$. Тогда будем иметь $(\overbrace{+\cdots+}^{n_{1}-1} \overbrace{-\cdots-}^{n_{2}} \overbrace{+\cdots+}^{n_{3}} \overbrace{-\cdots-}^{n_{4}}+)$. Далее переместим знак - из $n_{1}$-й позиции в 0-ю позицию с помошью $w_{3}=S_{N} S_{1} \ldots S_{n_{1}-2} S_{n_{1}-1}$ при $l\left(w_{3}\right)=n_{1}$, а знак переместим из $\left(n_{1}+n_{2}+n_{3}-1\right)$-й позиции в $\left(n_{1}+n_{2}\right)$-ю позицию с помощью $w_{4}=S_{n_{1}+n_{2}+1} \ldots S_{n_{1}+n_{2}+n_{3}}$ при $l\left(w_{4}\right)=n_{3}$. Таким образом, в результате перестановки $w=w_{4} w_{3} w_{2} w_{1}$ при $l(w)=N$ последовательность $\sigma$ возврашается в исходную. Заметим, что все последовательности на промежуточных шагах различны. Легко распространить эту процедуру и на общий случай.

Рассмотрим изменения знаков $a_{k}$ при переходе через сингулярности, определяемые с помощью $\tau_{k}\left(t_{*}\right)=0$ при некоторых $k$ и $t_{*} \in \mathbb{R}$. Из (3.19) тогда следует, что если $\tau_{k}(t) \sim$ $t-t_{*}$ вблизи $t=t_{*}$, то решения $a_{k}(t)$ и $b_{k}(t)$ вблизи $t=t_{*}$ имеют вид

$$
\begin{gathered}
a_{k}(t) \sim \frac{1}{\left(t-t_{*}\right)^{2}}, \quad a_{k-1}(t) \sim t-t_{*}, \quad a_{k+1}(t) \sim t-t_{*}, \\
b_{k}(t) \sim \frac{1}{t-t_{*}}, \quad b_{k+1}(t) \sim \frac{1}{t-t_{*}}
\end{gathered}
$$

и все остальные переменные $a_{j}$ и $b_{j}$ регулярны вблизи $t=t_{*}$. Заметим, что $a_{k}$ сохраняют тот же (отрицательный) знак до и после точки сингулярности, а $a_{k \pm 1}$ меняют свои знаки. Отсюда следует, что изменения знаков $a_{k \pm 1}$ при переходе через сингулярность $a_{k}$ соответствуют перестановкам $S_{k}$. Напомним, что $\epsilon_{k}=\operatorname{sign} a_{k}=\sigma_{k} \sigma_{k+1}$. Тогда лемма 4.3 подсказывает, что цепочка знаков $\sigma$, появляюшихся в периодических перестановках $w_{N}^{(\sigma)}$, снабжает метками цилиндры в каждом торе $\left(\mathbb{T}^{g}\right)_{j}$, и правило склейки дается знаковым представлением аффинной групшы Вейля $W$. Может оказаться удобным использование $\epsilon$-знака в качестве метки. Тогда для изменения знака мы имеем общую формулу в терминах расширенной матрицы Картана $\left(C_{i, j}\right)_{1 \leqslant i, j \leqslant N}$ для $A_{N-1}^{(1)}$ :

$$
S_{k}: \epsilon_{j} \longmapsto \epsilon_{j} \epsilon_{k}^{-C_{k, j}},
$$


которая была также получена в работе [12] для случая непериодической решетки Тоды. Суммируем все вьшесказанное в виде следуюшей гипотезы.

ГИПОТЕЗА 4.2. Каждый связный кусок (иилиндр) в аффинных частях изоспектрального множества уровня $Z_{\mathbb{R}}^{P}(\gamma)$ можно маркировать знаками $\left(\epsilon_{1}, \ldots, \epsilon_{N}\right)$, где $\epsilon_{k}=\operatorname{sign} a_{k}$, и они склеиваются друг с другом согласно действию әлемента аффинной группь Вейля $W$ длины $N$ на этих знаках (знаковое представление группь $W$ ).

Пример 4.2. $N=3$ периодическая решетка Тоды [13].

1. $m=2\left(N_{R}=3\right.$, три связных компоненты в $\left.\mathcal{R}_{\mathbb{R}}\right)$. Вешественная часть якобиана $\operatorname{Jac}(\mathcal{R})_{\mathbb{R}}$ состоит из четырех торов, а аффинная часть имеет вид

$$
\operatorname{Jac}(\mathcal{R})_{\mathbb{R}} \backslash \Theta \cong \mathcal{M}_{0}^{\circ} \sqcup \mathcal{M}_{1}^{\circ} \sqcup \mathcal{M}_{2}^{\circ},
$$

где

$$
\begin{aligned}
& \mathcal{M}_{2}^{\circ}=\left(\mathbb{T}^{2}\right)_{2}=\mathbb{T}^{2}, \\
& \mathcal{M}_{1}^{\circ}=\left(\mathbb{T}^{2} \backslash \Theta\right)_{1} \sqcup\left(\mathbb{T}^{2} \backslash \Theta\right)_{1}, \\
& \mathcal{M}_{0}^{\circ}=\left(\mathbb{T}^{2} \backslash \Theta\right)_{0}=\mathbb{R}^{2} \sqcup \mathbb{R}^{2} \sqcup \mathbb{R}^{2} .
\end{aligned}
$$

Каждый тор $\left(T^{2} \backslash \Theta\right)_{1}$ состоит из трех цилиндров $\mathbb{R} \times S^{1}$. Четыре тора $\mathbb{T}^{2}$, как компактифицированные множества уровня, маркированы знаками $\left(\epsilon_{1}, \epsilon_{2}, \epsilon_{3}\right)$, причем

$$
\prod_{k=1}^{3} \epsilon_{k}=1
$$

Например, тор $\mathbb{T}^{2}$ в $\mathcal{M}_{2}^{\circ}$ маркирован знаками $(+++)$, и каждая связная компонента в $\left(\mathbb{T}^{2} \backslash \Theta\right)_{j}$ для остальных $\mathcal{M}_{j}$ маркирована или $(+--)$, или $(-+-)$, или $(--+)$. Эти компоненты склеиваются друг с другом согласно действию $W$ на знаках:

$$
(+--) \stackrel{S_{2}}{\longrightarrow}(--+) \stackrel{S_{1}}{\longrightarrow}(-+-) \stackrel{S_{3}}{\longrightarrow}(+--) .
$$

2. $m=1\left(N_{R}=2\right)$. Вешественная часть якобиана $\operatorname{Jac}(\mathcal{R})_{\mathbb{R}}$ состоит из двух торов, а аффинная часть имеет вид

$$
\operatorname{Jac}(\mathcal{R})_{\mathbb{R}} \backslash \Theta \cong \mathcal{M}_{0}^{\circ} \sqcup \mathcal{M}_{1}^{\circ}
$$

где

$$
\begin{aligned}
& \mathcal{M}_{1}^{\circ}=\left(\mathbb{T}^{2} \backslash \Theta\right)_{1}=\left(\mathbb{R} \times S^{1}\right) \sqcup\left(\mathbb{R} \times S^{1}\right) \sqcup\left(\mathbb{R} \times S^{1}\right), \\
& \mathcal{M}_{0}^{\circ}=\left(\mathbb{T}^{2} \backslash \Theta\right)_{0}=\mathbb{R}^{2} \sqcup \mathbb{R}^{2} \sqcup \mathbb{R}^{2} .
\end{aligned}
$$

3. $m=0\left(N_{R}=1\right)$. Вещественная часть якобиана $\operatorname{Jac}(\mathcal{R})_{\mathbb{R}}$ является просто одним тором, а аффинная часть имеет вид

$$
\operatorname{Jac}(\mathcal{R})_{\mathbb{R}} \backslash \Theta \cong \mathcal{M}_{0}^{\circ}=\left(\mathbb{T}^{2} \backslash \Theta\right)_{0}=\mathbb{R}^{2} \sqcup \mathbb{R}^{2} \sqcup \mathbb{R}^{2} .
$$


Благодарности. Автор благодарен Н. Эрколани, Х. Флашка и Дж. Гиббонсу за ценные обсуждения и предложения, а также Л. Касиану, который выдвинул несколько полезных идей во время плодотворного сотрудничества на ранних стадиях данной работы. Работа была частично поддержана со стороны NSF (грант DMS0071523) и программы "Интегрируемые системы" в Институте математических наук Исаака Ньютона в период июль-сентябрь 2001 г.

\section{Список литературы}

[1] Y. Kodama, J. Ye. Physica D. 1998. V. 121. P. 89-108.

[2] M. Adler, P. van Moerbeke. Invent. Math. 1991. V. 103. P. 223-278.

[3] G. Weichold. Zeit. J. Math. Phys. 1883. V. 28. P. 321-351.

[4] M. Audin. Spinning Top. Cambridge: Cambridge Univ. Press, 1996; B. H. Gross, J. Harris. Ann. Sci. Ec. Norm. Sup. 1981. V. 14. P. 157-182.

[5] D. Mumford. Tata Lectures on Theta II. Progr. in Math. V.43. Boston: Birkhäuser, 1984.

[6] M. Toda. Theory of Nonlinear Lattices. New York: Springer, 1981.

[7] P. Vanhaecke. Math. Z. 1998. V. 227. P. 93-127.

[8] H. Farkas, I. Kra. Riemann surfaces. Graduate Texts in Math. V.71. New York: Springer, 1980.

[9] H. Flaschka, L. Haine. Math. Z. 1991. V. 208. P. 545-556.

[10] M. Adler, L. Haine, P. van Moerbeke. Math. Annal. 1993. V. 296. P. 1-33.

[11] S. Abenda, Yu. Fedorov. Acta Appl. Math. 2000. V. 60. P. 137-178.

[12] L. Casian, Y. Kodama. Toda lattice and toric varieties for real split semisimple Lie algebras. math-SG/9912021; Pacific J. Math. (in press).

[13] M. Audin. Ann. Inst. Fourier. 1994. V. 44. P. 1505-1517. 